ANNALES

POLONICI MATHEMATICI

$95.2(2009)$

\title{
Probability distribution solutions of a general linear equation of infinite order
}

\author{
by Tomasz Kochanek and Janusz Morawiec (Katowice)
}

\begin{abstract}
Let $(\Omega, \mathcal{A}, P)$ be a probability space and let $\tau: \mathbb{R} \times \Omega \rightarrow \mathbb{R}$ be strictly increasing and continuous with respect to the first variable, and $\mathcal{A}$-measurable with respect to the second variable. We obtain a partial characterization and a uniqueness-type result for solutions of the general linear equation

$$
F(x)=\int_{\Omega} F(\tau(x, \omega)) P(d \omega)
$$
\end{abstract}

in the class of probability distribution functions.

1. Introduction. In this paper we deal with the linear functional equation

$$
F(x)=\int_{\Omega} F(\tau(x, \omega)) P(d \omega) .
$$

Several particular cases of (1) appear in various areas of applications. For instance, in the case where $\tau(x, \omega)=x+\omega$ the corresponding equation, called the Integrated Cauchy Functional Equation, is of importance in probability theory (see [27], [28]). G. Choquet and J. Deny were the first to consider that version of (1) (see [3], [9]). The case $\tau(x, \omega)=\alpha x+\omega$ is closely connected with refinement equations (see [8], [15], [26]), which generate wavelets bases (see [4], [7], [20]) and splines (see [6], [19]). They are also fundamental to subdivision schemes (see [5], [10]). Equation (1) also appears in such areas of mathematics as iterated function systems (see [12], [14]), Markov chains (see [11], [21]) and perpetuities (see [13], [16], [29]).

For more information about results concerning equation (1) the reader is referred to the survey paper [1], and to [17], [18] for a complete theory of iterative functional equations.

2000 Mathematics Subject Classification: Primary 60E05, 39B12; Secondary 39B22, 45A05. Key words and phrases: linear functional equations, iterative functional equations, probability distribution solutions, extension of solutions, uniqueness of solutions. 
In the present paper we deal with the following problem: what can be said about uniqueness and properties of probability distribution (p.d.) solutions of (1) assuming only reasonable conditions on the given mapping $\tau$ ? We establish a uniqueness-type result which allows us to determine all p.d. solutions, provided we know all continuous p.d. solutions satisfying some special boundary conditions.

2. Preliminaries. Throughout the paper, $(\Omega, \mathcal{A}, P)$ is a probability space and $\tau: \mathbb{R} \times \Omega \rightarrow \mathbb{R}$ is a mapping such that for every $x \in \mathbb{R}$ the function $\tau(x, \cdot)$ is $\mathcal{A}$-measurable, and for every $\omega \in \Omega$ the function $\tau(\cdot, \omega)$ is strictly increasing and continuous.

We are interested in the following two classes of solutions of (1): $\mathcal{I}:=\{F: \mathbb{R} \rightarrow[0,1] \mid F$ is a weakly increasing solution of (1) such that $F(-\infty):=\lim _{x \rightarrow-\infty} F(x)=0$ and $\left.F(+\infty):=\lim _{x \rightarrow+\infty} F(x)=1\right\}$, $\mathcal{C}:=\{F \in \mathcal{I}: F$ is continuous $\}$.

It will be convenient to consider equation (1) in a more general situation. If $I \subset \mathbb{R}$ is an interval and $\sigma: I \times \Omega \rightarrow I$ is a mapping which is weakly increasing and continuous with respect to the first variable, and $\mathcal{A}$-measurable with respect to the second variable, then we rewrite (1) as

$$
F(x)=\int_{\Omega} F(\sigma(x, \omega)) P(d \omega) .
$$

We denote by $\mathcal{C}_{\sigma}(I)$ the class of all continuous and weakly increasing solutions $F: I \rightarrow \mathbb{R}$ of (2), and put

$$
\mathcal{C}_{\sigma}^{0}(I)=\left\{F \in \mathcal{C}_{\sigma}(I): \lim _{x \rightarrow \inf I} F(x)=0 \text { and } \lim _{x \rightarrow \sup I} F(x)=1\right\} .
$$

We say that a subset $S$ of $I$ is $\sigma$-invariant if $S \neq \emptyset$ and for every $x \in S$ we have $\sigma(x, \omega) \in S$ for almost all $\omega \in \Omega$.

Given a $\sigma$-invariant subinterval $J$ of $I$ define a mapping $\sigma_{J}: J \times \Omega \rightarrow J$ by putting $\sigma_{J}(x, \omega)=\sigma(x, \omega)$ if $\sigma(x, \omega) \in J$, and $\sigma_{J}(x, \omega)=0$ otherwise. It is evident that for every function $F: I \rightarrow[0,1]$ we have $\left.F\right|_{J} \in \mathcal{C}_{\sigma_{J}}^{0}(J)$ if and only if $F \in \mathcal{C}_{\sigma}^{0}(I), \lim _{x \rightarrow \inf J} F(x)=0$ and $\lim _{x \rightarrow \sup J} F(x)=1$. Therefore, for every $\sigma$-invariant subinterval $J$ of $I$ we will use the symbol $\mathcal{C}_{\sigma}^{0}(J)$ instead of $\mathcal{C}_{\sigma_{J}}^{0}(J)$.

Define

$$
\mathbf{E}_{\sigma}=\{x \in I: \sigma(x, \omega)=x \text { for almost all } \omega \in \Omega\} .
$$

Clearly, $\mathbf{E}_{\sigma}$ is closed. Let $\mathcal{U}_{\sigma}$ be the family of all open components of $I \backslash \mathbf{E}_{\sigma}$. Note that each such component is a $\sigma$-invariant interval disjoint from $\mathbf{E}_{\sigma}$.

We now quote the main result from [24] which is the first step in determining the class $\mathcal{I}$ (cf. also [23] where a result of similar type was established in a very particular case of (1)). 
Theorem 1 (see [24, Theorem 2]).

(i) If $\mathbf{E}_{\tau}=\emptyset$, then $\mathcal{C}=\mathcal{I}$.

(ii) If $\mathbf{E}_{\tau} \neq \emptyset$, then $\mathcal{C} \subsetneq \mathcal{I}$. Moreover, a function $F: \mathbb{R} \rightarrow[0,1]$ belongs to $\mathcal{I}$ if and only if it is weakly increasing, $F(-\infty)=0, F(+\infty)=1$ and on every component $J \in \mathcal{U}_{\tau}$, either $F$ is constant or the function given by

$$
F_{J}(x)=\frac{F(x)-F(\inf J)}{F(\sup J)-F(\inf J)}
$$

belongs to $\mathcal{C}_{\tau}^{0}(J)$.

We see that p.d. solutions of (1) may be defined arbitrarily on $\mathbf{E}_{\tau}$ (they just have to meet the requirements in Theorem 1(ii)), whereas their behaviour on every component $J \in \mathcal{U}_{\tau}$ is determined by functions from $\mathcal{C}_{\tau}^{0}(J)$. It turns out that all functions belonging to that class may be described by functions from $\mathcal{C}_{\sigma}^{0}(\mathbb{R})$ with a suitable $\sigma: \mathbb{R} \times \Omega \rightarrow \mathbb{R}$ satisfying $\mathbf{E}_{\sigma}=\emptyset$. To see this, fix $J \in \mathcal{U}_{\tau}$, any increasing homeomorphism $\phi_{J}: \mathbb{R} \rightarrow J$ and define

$$
\sigma(\cdot, \omega)=\phi_{J}^{-1} \circ \tau(\cdot, \omega) \circ \phi_{J} .
$$

Plainly, $\sigma$ is strictly increasing and continuous with respect to the first variable, and $\mathcal{A}$-measurable with respect to the second. A simple calculation shows that $F_{J} \in \mathcal{C}_{\tau}^{0}(J)$ if and only if $F_{J} \circ \phi_{J} \in \mathcal{C}_{\sigma}^{0}(\mathbb{R})$. Moreover, $\tau$-invariant subsets $S \subset J$ are in one-to-one correspondence with $\sigma$-invariant sets $\phi_{J}^{-1}(S)$. In particular, since $J \cap \mathbf{E}_{\tau}=\emptyset$, we have $\mathbf{E}_{\sigma}=\emptyset$.

The above argument, jointly with Theorem 1, justifies the assumption $\mathbf{E}_{\tau}=\emptyset$, which we will adopt from now on.

In Section 3 we prove the main result of this paper. In Section 4 we show how it can be used to describe solutions from the class $\mathcal{I}$ in terms of solutions from a very special subclass (see Corollary 2). We finish the paper with an example, included in Section 5, which demonstrates an application of our results.

\section{Uniqueness-type theorem. Let}

$$
\mathcal{S}_{\sigma}=\{S \subset I: S \text { is a minimal compact } \sigma \text {-invariant interval }\} \text {. }
$$

The main result of this paper reads as follows.

Theorem 2. Assume $\mathbf{E}_{\tau}=\emptyset$. Every $F \in \mathcal{I}$ is constant on each interval from $\mathcal{S}_{\tau}$. Moreover, for every $f: \mathcal{S}_{\tau} \rightarrow[0,1]$ there is at most one $F \in \mathcal{I}$ such that $\left.F\right|_{I}=f(I)$ for all $I \in \mathcal{S}_{\tau}$.

Let us stress that $\mathcal{S}_{\tau}=\emptyset$ may happen. In such a case (1) has at most one solution in the class of all p.d. functions. Of course, the "monotonicity" of the function $f$ is essential to produce a p.d. solution $F$. 
Proof. For transparency we divide the proof into several parts.

Claim 1. It is enough to prove the assertion of Theorem 2 under the assumption that $F$ is a continuous p.d. function.

This follows immediately from assertion (i) of Theorem 1.

In Claims 2-5 we constantly assume the following: $-\infty \leq \alpha<\beta \leq+\infty$, $I=\operatorname{cl}(\alpha, \beta)$ (here and below, cl stands for closure in $\mathbb{R}$ ), and $\sigma: I \times \Omega \rightarrow I$ is a mapping which is weakly increasing and continuous with respect to the first variable, $\mathcal{A}$-measurable with respect to the second variable, and such that $\mathbf{E}_{\sigma}=\emptyset$. We recall that $F( \pm \infty)$ always stands for $\lim _{x \rightarrow \pm \infty} F(x)$.

Claim 2. If there are distinct $F, G \in \mathcal{C}_{\sigma}(I)$ such that $F(\alpha)=G(\alpha)$ and $F(\beta)=G(\beta)$, then $\mathcal{S}_{\sigma}$ is non-void.

Put

$$
\begin{aligned}
M & =\sup \{|F(x)-G(x)|: x \in I\}>0, \\
S & =\{x \in I:|F(x)-G(x)|=M\}, \\
S_{n} & =\{x \in I:|F(x)-G(x)| \leq M-1 / n\} \quad \text { for } n \in \mathbb{N} .
\end{aligned}
$$

Evidently, $S$ is a non-void and compact subset of $I$, and $I \backslash S=\bigcup_{n \in \mathbb{N}} S_{n}$. Let

$$
N=\{x \in I: P(\sigma(x, \omega) \in S)=1\} .
$$

Assume that there exists $x_{0} \in I \backslash N$. This means that $P\left(\sigma\left(x_{0}, \omega\right) \notin S\right)>0$, and thus

$$
\alpha_{0}:=P\left(\sigma\left(x_{0}, \omega\right) \in S_{n_{0}}\right)>0
$$

for sufficiently large $n_{0} \in \mathbb{N}$. Set

$$
\Omega_{0}=\left\{\omega \in \Omega: \sigma\left(x_{0}, \omega\right) \in S_{n_{0}}\right\} .
$$

Then equation (2) implies

$$
\begin{aligned}
\left|F\left(x_{0}\right)-G\left(x_{0}\right)\right| & \leq \int_{\Omega}\left|F\left(\sigma\left(x_{0}, \omega\right)\right)-G\left(\sigma\left(x_{0}, \omega\right)\right)\right| P(d \omega) \\
& =\int_{\Omega_{0}}+\int_{\Omega \backslash \Omega_{0}} \leq \alpha_{0}\left(M-\frac{1}{n_{0}}\right)+\left(1-\alpha_{0}\right) M<M,
\end{aligned}
$$

which shows that $x_{0} \notin S$. We infer that $S \subset N$, hence $S$ is $\sigma$-invariant.

If $s_{1}:=\inf S$ and $s_{2}:=\sup S$, then $\sigma\left(s_{1}, \omega\right) \geq s_{1}$ and $\sigma\left(s_{2}, \omega\right) \leq s_{2}$ for almost all $\omega \in \Omega$, which, jointly with monotonicity of $\sigma$, implies that the interval $\left[s_{1}, s_{2}\right]$ is $\sigma$-invariant.

It remains to apply the Zorn-Kuratowski lemma to the family

$$
\{S \subset I: S \text { is a compact and } \sigma \text {-invariant interval }\} \text {. }
$$

From now on $\widetilde{I}$ stands for an element of $\mathcal{S}_{\sigma}$. 
Claim 3. Define $\phi: \widetilde{I} \rightarrow \widetilde{I}$ by

$$
\phi(x)=\sup \{y \in \widetilde{I}: P(\sigma(x, \omega) \geq y)>0\} .
$$

Then:

(i) $\phi$ is weakly increasing and left-continuous;

(ii) for every $x \in[\inf \widetilde{I}$, sup $\widetilde{I})$ we have $x<\phi(x)$.

The fact that $\phi$ is weakly increasing is an easy consequence of the fact that $\sigma$ weakly increases as a function of the first variable.

For the left-continuity suppose, on the contrary, that $x_{0} \in \widetilde{I}$ and there exists a strictly increasing sequence $\left(x_{n}\right)_{n \in \mathbb{N}}$ in $\widetilde{I}$ such that

$$
\lim _{n \rightarrow \infty} x_{n}=x_{0} \quad \text { and } \quad \gamma:=\lim _{n \rightarrow \infty} \phi\left(x_{n}\right)<\phi\left(x_{0}\right) .
$$

Choose any numbers $\nu, \xi$ such that $\gamma<\nu<\xi<\phi\left(x_{0}\right)$. By the definition of $\phi$, the set

$$
C:=\left\{\omega \in \Omega: \sigma\left(x_{0}, \omega\right) \geq \xi\right\}
$$

has a positive measure. Let

$$
C_{n}=\left\{\omega \in C: \sigma\left(x_{n}, \omega\right) \geq \nu\right\} \quad \text { for } n \in \mathbb{N} .
$$

The continuity of $\sigma$ as a function of the first variable yields

$$
\bigcup_{n \in \mathbb{N}} C_{n}=C \text {. }
$$

Since $\phi\left(x_{n}\right)<\nu$, we have $P\left(C_{n}\right)=0$ for $n \in \mathbb{N}$, hence $P(C)=0$; a contradiction.

Finally, suppose that $\phi(x) \leq x$ for some $x \in[\inf \widetilde{I}, \sup \widetilde{I})$. Then by the definition of $\phi$, no $y \in \widetilde{I}$ with $P(\sigma(x, \omega) \geq y)>0$ exceeds $x$. Hence

$$
P(\sigma(x, \omega)>x) \leq \sum_{n \in \mathbb{N}} P(\sigma(x, \omega) \geq x+1 / n)=0,
$$

which means that $\sigma(x, \omega) \leq x$ for almost all $\omega \in \Omega$. However, the monotonicity of $\sigma$ with respect to the first variable would then imply that the interval $[\inf \widetilde{I}, x]$ is $\sigma$-invariant and $[\inf \widetilde{I}, x] \subsetneq \widetilde{I}$, which contradicts the fact that $\widetilde{I}$ is minimal.

Claim 4. Define $\psi: \widetilde{I} \rightarrow \widetilde{I}$ by

$$
\psi(x)=\frac{1}{2}(x+\phi(x)) .
$$

Let $\psi^{n}$ stand for the $n$th iterate of $\psi$. Then:

(i) $\psi^{n}(\inf \widetilde{I})<\sup \widetilde{I}$ for $n \geq 0$;

(ii) the sequence $\left(\psi^{n}(\inf \widetilde{I})\right)_{n \geq 0}$ is strictly increasing;

(iii) $\lim _{n \rightarrow \infty} \psi^{n}(\inf \widetilde{I})=\sup \widetilde{I}$. 
Inequality (i) follows directly from the formula of $\psi$ and the fact that the interval $\widetilde{I}$ is non-degenerate (which is a consequence of the assumption $\left.\mathbf{E}_{\sigma}=\emptyset\right)$.

With the aid of assertion (i) and Claim 3(i) we easily obtain (ii).

For the proof of (iii) set $\gamma=\lim _{n \rightarrow \infty} \psi^{n}(\inf \widetilde{I})$ and suppose that $\gamma<$ $\sup \widetilde{I}$. From the equality

$$
\psi^{n+1}(\inf \widetilde{I})=\frac{1}{2}\left(\psi^{n}(\inf \widetilde{I})+\phi\left(\psi^{n}(\inf \widetilde{I})\right)\right) \quad \text { for } n \in \mathbb{N}
$$

we get $\lim _{n \rightarrow \infty} \phi\left(\psi^{n}(\inf \widetilde{I})\right)=\gamma$. However, by (ii) and Claim 3(i), the last limit equals $\phi(\gamma)$ and we obtain $\phi(\gamma)=\gamma$, which contradicts Claim 3(ii).

Claim 5. If $F \in \mathcal{C}_{\sigma}(I)$, then $F$ is constant on $\widetilde{I}$.

For $n \geq 0$ let $J_{n}=\left[\psi^{n}(\inf \widetilde{I}), \psi^{n+1}(\inf \widetilde{I})\right]$. In the light of Claim 4, it suffices to prove that $\left.F\right|_{J_{n}}$ is constant for every $n \geq 0$. Put $\xi_{n}=\psi^{n}(\inf \widetilde{I})$. Assume inductively that $F\left(\xi_{n}\right)=F(\inf \widetilde{I}$ ) (which is trivial for $n=0$ ) and fix $x \in J_{n}=\left[\xi_{n}, \psi\left(\xi_{n}\right)\right]$. By Claims 3(ii) and 4(i), we infer that $\psi\left(\xi_{n}\right)<\phi\left(\xi_{n}\right)$, hence the set

$$
\Omega_{0}:=\left\{\omega \in \Omega: \sigma\left(\xi_{n}, \omega\right) \geq x\right\}
$$

is of a positive probability $\alpha_{0}$. Since $F \in \mathcal{C}_{\sigma}(I)$ and $\sigma\left(\xi_{n}, \omega\right) \geq \inf \widetilde{I}$ for almost all $\omega \in \Omega$, we have

$$
\begin{aligned}
F(\inf \widetilde{I}) & =F\left(\xi_{n}\right)=\int_{\Omega} F\left(\sigma\left(\xi_{n}, \omega\right)\right) P(d \omega)=\int_{\Omega_{0}}+\int_{\Omega \backslash \Omega_{0}} \\
& \geq \alpha_{0} F(x)+\left(1-\alpha_{0}\right) F(\inf \widetilde{I}) .
\end{aligned}
$$

This implies that $F(x) \leq F(\inf \widetilde{I})$, thus $F(x)=F(\inf \widetilde{I})$.

Before we proceed with the proof, let us introduce some notation. If $S \subset \mathbb{R}$ is a $\tau$-invariant interval such that every $F \in \mathcal{C}_{\tau}(\mathbb{R})$ is constant on $S$, let $\kappa(S)$ denote a maximal $\tau$-invariant interval such that $S \subset \kappa(S)$ and every $F \in \mathcal{C}_{\tau}(\mathbb{R})$ is constant on $\kappa(S)$. Obviously, such an interval exists, and the continuity of functions from $\mathcal{C}_{\tau}(\mathbb{R})$ and of $\tau(\cdot, \omega)$ for $\omega \in \Omega$ implies that it is a closed interval. By Claim 5 (applied for $I=\mathbb{R}$ and $\sigma=\tau$ ), the symbol $\kappa(S)$ makes sense for every $S \in \mathcal{S}_{\tau}$. Define

$\mathcal{M}=\{J \subset \mathbb{R}: J$ is a maximal $\tau$-invariant interval such that every $F \in \mathcal{C}_{\tau}(\mathbb{R})$ is constant on $\left.J\right\}$.

The families $\mathcal{S}_{\tau}, \kappa\left(\mathcal{S}_{\tau}\right), \mathcal{M}$ each consist of pairwise disjoint non-degenerate closed intervals.

Claim 6. We have:

(i) $\kappa\left(\mathcal{S}_{\tau}\right) \subset \mathcal{M}$;

(ii) $\{J \in \mathcal{M}: J$ is compact $\} \subset \kappa\left(\mathcal{S}_{\tau}\right)$. 
The first assertion is clear. For the second, observe that if $J \in \mathcal{M}$ is compact, then there is $S \in \mathcal{S}_{\tau}$ with $S \subset J$. Plainly, $\kappa(S)=J$, so $J \in \kappa\left(\mathcal{S}_{\tau}\right)$.

Claim 7. The set $\cup \mathcal{M}$ is closed.

Suppose that there is $x_{0} \in(\operatorname{cl} \cup \mathcal{M}) \backslash \cup \mathcal{M}$. Then there exists either an increasing sequence of right end-points of intervals from $\mathcal{M}$ which converges to $x_{0}$, or a decreasing sequence of left end-points of intervals from $\mathcal{M}$ which converges to $x_{0}$. Without loss of generality, assume that the latter case holds true and let $\left(I_{n}\right)_{n \in \mathbb{N}}$ be a sequence of intervals from $\mathcal{M}$ such that $\inf I_{n+1}<\sup I_{n+1}<\inf I_{n}$ for $n \in \mathbb{N}$ and

$$
\lim _{n \rightarrow \infty} \inf I_{n}=x_{0}=\lim _{n \rightarrow \infty} \sup I_{n} .
$$

Since all the intervals $I_{n}$ are $\tau$-invariant, we infer that

$$
P\left(\tau\left(\inf I_{n}, \omega\right) \geq \inf I_{n}\right)=1 \quad \text { and } \quad P\left(\tau\left(\sup I_{n}, \omega\right) \leq \sup I_{n}\right)=1,
$$

hence $\tau\left(x_{0}, \omega\right)=x_{0}$ for almost all $\omega \in \Omega$, contrary to the fact that $\mathbf{E}_{\tau}=\emptyset$.

Claim 8. For every $g: \mathcal{M} \rightarrow[0,1]$ there exists at most one $F \in \mathcal{C}$ such that $\left.F\right|_{I}=g(I)$ for all $I \in \mathcal{M}$.

Suppose $F, G \in \mathcal{C}, F \neq G$ and $\left.F\right|_{I}=g(I)=\left.G\right|_{I}$ for all $I \in \mathcal{M}$. By Claim 7 , the set $\mathbb{R} \backslash \bigcup \mathcal{M}$ is open. Choose any of its components, $(\alpha, \beta)$, on which $F$ and $G$ do not coincide.

Let $I=\operatorname{cl}(\alpha, \beta)$ and $\widetilde{F}=\left.F\right|_{I}, \widetilde{G}=\left.G\right|_{I}$. It is obvious that $\widetilde{F}$ and $\widetilde{G}$ are continuous, weakly increasing and $\widetilde{F}(\alpha)=\widetilde{G}(\alpha), \widetilde{F}(\beta)=\widetilde{G}(\beta)$. Define a mapping $\sigma: I \times \Omega \rightarrow I$ as follows. For every $x \in I$ and $\omega \in \Omega$ put

$$
\sigma(x, \omega)= \begin{cases}\tau(x, \omega) & \text { if } \tau(x, \omega) \in I, \\ \alpha & \text { if } \tau(x, \omega)<\alpha, \\ \beta & \text { if } \tau(x, \omega)>\beta\end{cases}
$$

It is easily seen that $\sigma$ is weakly increasing and continuous with respect to the first variable, and $\mathcal{A}$-measurable with respect to the second. Moreover, $\mathbf{E}_{\sigma}=\emptyset$. Now, we are going to verify that $\widetilde{F}$ and $\widetilde{G}$ satisfy (2).

Fix $x \in I$. Assume that $\beta<+\infty$; then $\beta \in \bigcup \mathcal{M}$, so it is a lower bound of one of the intervals from $\mathcal{M}$, say $I_{t_{0}}=\operatorname{cl}\left[\beta, \sup I_{t_{0}}\right)$. This implies that

and therefore

$$
P\left(\tau(\beta, \omega) \leq \sup I_{t_{0}}\right)=1,
$$

$$
P\left(\tau(x, \omega) \leq \sup I_{t_{0}}\right)=1 .
$$

Directly from the definition of $\sigma$ we infer that

$$
\int_{\{\tau(x, \omega)>\beta\}} \widetilde{F}(\sigma(x, \omega)) P(d \omega)=P(\tau(x, \omega)>\beta) \cdot F(\beta) .
$$


Condition (5) implies that $\tau(x, \omega) \in I_{t_{0}}$ for almost all $\omega \in\{\tau(x, \omega)>\beta\}$. Since $F$ is constant on the interval $I_{t_{0}}$, we have

$$
\int_{\{\tau(x, \omega)>\beta\}} F(\tau(x, \omega)) P(d \omega)=P(\tau(x, \omega)>\beta) \cdot F(\beta) .
$$

Hence

$$
\int_{\{\tau(x, \omega)>\beta\}} \widetilde{F}(\sigma(x, \omega)) P(d \omega)=\int_{\{\tau(x, \omega)>\beta\}} F(\tau(x, \omega)) P(d \omega) .
$$

In the case where $\beta=+\infty$ the above equality is trivial. Analogously we show that

$$
\int_{\{\tau(x, \omega)<\alpha\}} \widetilde{F}(\sigma(x, \omega)) P(d \omega)=\int_{\{\tau(x, \omega)<\alpha\}} F(\tau(x, \omega)) P(d \omega) .
$$

Plainly,

$$
\int_{\{\tau(x, \omega) \in I\}} \widetilde{F}(\sigma(x, \omega)) P(d \omega)=\int_{\{\tau(x, \omega) \in I\}} F(\tau(x, \omega)) P(d \omega) .
$$

Summing up equations (6)-(8) we obtain

$$
\int_{\Omega} \widetilde{F}(\sigma(x, \omega)) P(d \omega)=\int_{\Omega} F(\tau(x, \omega)) P(d \omega),
$$

which shows that $\widetilde{F}$ (and $\widetilde{G}$ as well) satisfies (2). Consequently, $\widetilde{F}, \widetilde{G} \in \mathcal{C}_{\sigma}(I)$.

By Claim 2, there exists $\widetilde{I} \subset I$ such that $\widetilde{I} \in \mathcal{S}_{\sigma}$. We have just proved that for every $F \in \mathcal{C}$ its restriction $\widetilde{F}=\left.F\right|_{I}$ belongs to $\mathcal{C}_{\sigma}(I)$, thus Claim 5 shows that $\widetilde{F}$, and so $F$ itself, is constant on $\widetilde{I}$. Consequently, the symbol $\kappa(\widetilde{I})$ makes sense.

Fix $x \in \widetilde{I}$. Since $\widetilde{I}$ is $\sigma$-invariant, we have

$$
\begin{aligned}
& P(\sigma(x, \omega) \leq \beta) \geq P(\sigma(\sup \widetilde{I}, \omega) \leq \beta) \geq P(\sigma(\sup \widetilde{I}, \omega) \leq \sup \widetilde{I})=1, \\
& P(\sigma(x, \omega) \geq \alpha) \geq P(\sigma(\inf \widetilde{I}, \omega) \geq \alpha) \geq P(\sigma(\inf \widetilde{I}, \omega) \geq \inf \widetilde{I})=1 .
\end{aligned}
$$

Hence for all $x \in \widetilde{I}$ and almost all $\omega \in \Omega$ we have $\sigma(x, \omega)=\tau(x, \omega)$, which implies that $\widetilde{I}$ is $\tau$-invariant, so $\kappa(\widetilde{I}) \in \mathcal{M}$, a contradiction.

Claim 9. For every $f: \mathcal{S}_{\tau} \rightarrow[0,1]$ there exists at most one $F \in \mathcal{C}$ such that $\left.F\right|_{I}=f(I)$ for all $I \in \mathcal{S}_{\tau}$.

Suppose that there is $F \in \mathcal{C}$ satisfying $\left.F\right|_{I}=f(I)$ for all $I \in \mathcal{S}_{\tau}$. Define $g: \mathcal{M} \rightarrow[0,1]$ by

$$
g(J)= \begin{cases}f(I) & \text { if } J=\kappa(I) \text { for some } I \in \mathcal{S}_{\tau}, \\ 0 & \text { if } J=(-\infty, a] \text { for some } a \in \mathbb{R}, \\ 1 & \text { if } J=[b,+\infty) \text { for some } b \in \mathbb{R} .\end{cases}
$$


In view of Claim 6 and the fact that at least one solution of (1) exists, the definition is correct. Of course, $\left.F\right|_{J}=g(J)$ for all $J \in \mathcal{M}$ and Claim 8 implies that $F$ is uniquely determined.

This completes the proof of Theorem 2 .

4. Concluding remarks. The following is an immediate consequence of Theorem 2.

COROLlaRY 1. If $\mathbf{E}_{\tau}=\emptyset$ and there exists a strictly increasing function $F \in \mathcal{I}$, then $\mathcal{I}=\{F\}$.

Observe that a proof similar to that of Claim 7 shows that $\bigcup \mathcal{S}_{\tau}$ is closed. Consider any component $J$ of the open set $\mathbb{R} \backslash \cup \mathcal{S}_{\tau}$. Let $\widetilde{F}_{J}$ stand for a function from $\mathcal{C}$ such that

$$
\lim _{x \rightarrow \inf J} \widetilde{F}_{J}(x)=0 \text { and } \lim _{x \rightarrow \sup J} \widetilde{F}_{J}(x)=1,
$$

provided it exists. By Theorem 2, such a function is then unique. The following corollary is the next step in reducing the investigation of the class $\mathcal{I}$ to some special situations. In fact, now we may focus on solutions $\widetilde{F}_{J}$ such that $\widetilde{F}_{J}(x)=0$ for $x \in(-\infty$, inf $J]$ and $\widetilde{F}_{J}(x)=1$ for $x \in[\sup J,+\infty)$.

Corollary 2. Assume $\mathbf{E}_{\tau}=\emptyset$. A function $F: \mathbb{R} \rightarrow[0,1]$ belongs to $\mathcal{I}$ if and only if it is weakly increasing, continuous, $F(-\infty)=0, F(+\infty)=1$, $\left.F\right|_{I}$ is constant for all $I \in \mathcal{S}_{\tau}$, and on every component $J$ of $\mathbb{R} \backslash \bigcup \mathcal{S}_{\tau}$ it is either constant or expressed by (3), where $\widetilde{F}_{J} \in \mathcal{C}$ satisfies (9) and is uniquely determined.

In the case where the component $J$ is bounded one can try to apply known results in order to get the existence of $\widetilde{F}_{J}$. One of such tools could be Corollary 1 from [22], where [0,1] plays the role of $\mathrm{cl} J$; see also [2].

Remark 1. Assume $\mathbf{E}_{\tau}=\emptyset$. Then $\mathcal{I} \neq \emptyset$ if and only if there exists at least one function $\widetilde{F}_{J} \in \mathcal{C}$ satisfying (9) for some component $J$ of $\mathbb{R} \backslash \bigcup \mathcal{S}_{\tau}$.

Proof. Sufficiency is clear. Now suppose that $F \in \mathcal{I}$, but no $\widetilde{F}_{J}$ exists. Then, since $F$ is continuous, we have

$$
(0,1)=F(\mathbb{R}) \backslash\{0,1\} \subset F\left(\bigcup \mathcal{S}_{\tau}\right) .
$$

However, the last set is countable, a contradiction.

REMARK 2. Assume $\mathbf{E}_{\tau}=\emptyset$. If $S$ is a $\tau$-invariant half-line disjoint from $\bigcup \mathcal{S}_{\tau}$, then every $F \in \mathcal{C}$ is constant on $S$.

Proof. If $S=[b,+\infty)$ for some $b \in \mathbb{R}$, one can verify that all arguments in Claims 3-5 work with $\widetilde{I}$ replaced by $S$. If $S=(-\infty, a]$ for some $a \in \mathbb{R}$, the proof runs analogously. One has to change sup to inf in the formula defining $\phi$. 
5. Example. We now demonstrate how Corollary 2, jointly with already known results, works in the specific case where

$$
\tau_{1}(x):=\left\{\begin{array}{ll}
x & \text { if } x \in(-\infty, 0), \\
3 x & \text { if } x \in\left[0, \frac{1}{3}\right), \\
\frac{3}{5} x+\frac{4}{5} & \text { if } x \in\left[\frac{1}{3}, 2\right), \\
2 x-2 & \text { if } x \in[2, \infty),
\end{array} \quad \tau_{2}(x):= \begin{cases}\frac{3}{5} x-\frac{2}{5} & \text { if } x \in\left(-\infty, \frac{2}{3}\right), \\
3 x-2 & \text { if } x \in\left[\frac{2}{3}, 1\right), \\
\frac{2}{3} x+\frac{1}{3} & \text { if } x \in\left[1, \frac{5}{2}\right), \\
2 x-3 & \text { if } x \in\left[\frac{5}{2}, \infty\right),\end{cases}\right.
$$

and the indices 1,2 are chosen with probability $1 / 2$.

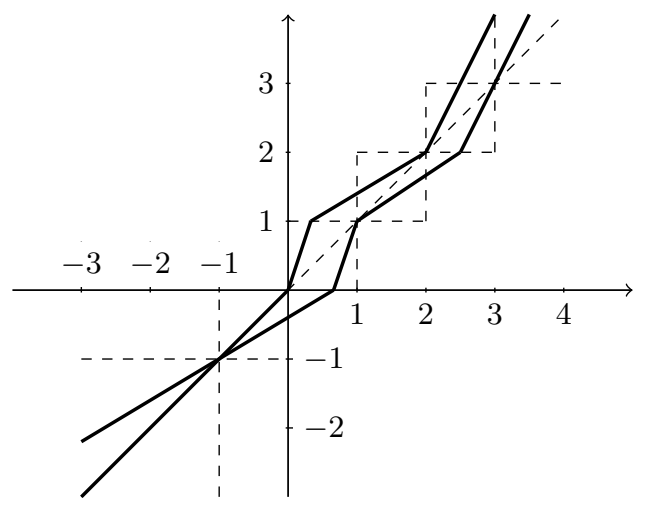

Fig. 1

In this case $\mathbf{E}_{\tau}=\{-1\}$ (see Figure 1) and, by Theorem 1(ii), $\mathcal{I} \neq \emptyset$ and we have to consider equation (1) separately on $(-\infty,-1)$ and on $(-1,+\infty)$. Fix $F \in \mathcal{I}$. The value $F(-1)$ may be an arbitrary number $a \in[0,1]$, and -1 is the only possible point of discontinuity, by Theorem 1(ii). The next remark shows that $\left.F\right|_{(-\infty,-1)}=0$.

REMARK 3. Assume $\mathbf{E}_{\tau}=\emptyset$. If either $\tau(x, \omega) \leq x$ for all $x \in \mathbb{R}$ and almost all $\omega \in \Omega$, or $\tau(x, \omega) \geq x$ for all $x \in \mathbb{R}$ and almost all $\omega \in \Omega$, then $\mathcal{I}=\emptyset$.

Proof. This follows from Remark 2. Indeed, in the first case every halfline $(-\infty, a]$ with $a \in \mathbb{R}$ is $\tau$-invariant, whereas in the second case every half-line $[b,+\infty)$ with $b \in \mathbb{R}$ is $\tau$-invariant. Plainly, $\mathcal{S}_{\tau}=\emptyset$.

Observe that $\mathcal{S}_{\tau}=\{[1,2]\}$, so Corollary 2 implies that $\left.F\right|_{[1,2]}$ is constant, say $c$ with $a \leq c \leq 1$. Since both $(-1,0]$ and $[3,+\infty)$ are $\tau$-invariant, Remark 3 yields $\left.F\right|_{[3,+\infty)}=1$ and $\left.F\right|_{(-1,0]}=b$ with $a \leq b \leq c$. Finally, according to [25] we infer that $F$ is the classical Cantor function on $[0,1]$ and an affine function on $[2,3]$.

Consequently, any solution $F \in \mathcal{I}$ depends on three parameters $0 \leq a \leq$ $b \leq c \leq 1$ and its graph looks like the one in Figure 2. 


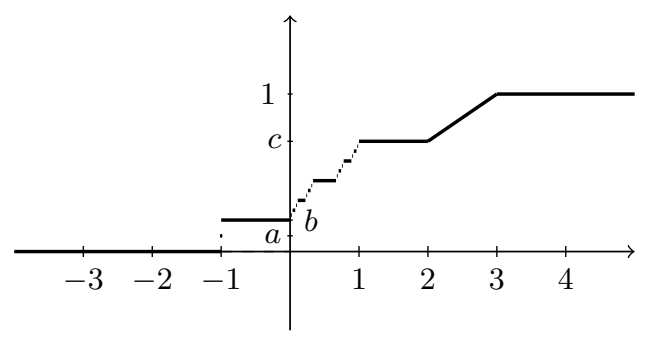

Fig. 2

Acknowledgements. This research was supported by the Silesian University Mathematics Department (Functional Equations on Abstract Structures program - the first author, and Functional Equations program - the second author).

\section{References}

[1] K. Baron and W. Jarczyk, Recent results on functional equations in a single variable, perspectives and open problems, Aequationes Math. 61 (2001), 1-48.

[2] - - - Random-valued functions and iterative functional equations, ibid. 67 (2004), 140-153.

[3] G. Choquet et J. Deny, Sur l'équation de convolution $\mu=\mu * \sigma$, C. R. Acad. Sci. Paris 250 (1960), 799-801.

[4] C. K. Chui and X. Shi, Continuous two-scale equations and dyadic wavelets, Adv. Comput. Math. 2 (1994), 185-213.

[5] W. Dahmen and C. A. Micchelli, Continuous refinement equations and subdivision, ibid. 1 (1993), 1-37.

[6] X. R. Dai, D. J. Feng and Y. Wang, Classification of refinable splines, Constr. Approx. 24 (2006), 187-200.

[7] I. Daubechies, Ten Lectures on Wavelets, CBMS-NSF Reg. Conf. Ser. Appl. Math. 61, SIAM, Philadelphia, 1992.

[8] A. Deliu and M. C. Spruill, Existence results for refinement equations, Aequationes Math. 59 (2000), 20-37.

[9] J. Deny, Sur l'équation de convolution, in: Séminaire de Théorie du Potentiel: 4e Année 1959/60, Secrétariat Math., Paris, 1961.

[10] G. Derfel, N. Dyn and D. Levin, Generalized refinement equations and subdivision processes, J. Approx. Theory 80 (1995), 272-297.

[11] L. E. Dubins and D. A. Freedman, Invariant properties for certain Markov processes, Ann. Math. Statist. 37 (1966), 837-848.

[12] K. J. Falconer, Fractal Geometry. Mathematical Foundations and Applications, Wiley, Chichester, 1990.

[13] A. K. Grincevičjus, On the continuity of the distribution of a sum of dependent variables connected with independent walks on lines, Teor. Veroyatnost. i Primenen. 19 (1974), 163-168 (in Russian); English transl.: Theory Probab. Appl. 19 (1974), $163-168$.

[14] J. E. Hutchinson, Fractals and self-similarity, Indiana Univ. Math. J. 30 (1981), 713-747. 
[15] R. Kapica and J. Morawiec, Probability distribution functions of the Grincevičjus series, J. Math. Anal. Appl. 342 (2008), 1380-1387.

[16] H. Kesten, Random difference equations and renewal theory for products of random matrices, Acta Math. 131 (1973), 207-248.

[17] M. Kuczma, Functional Equations in a Single Variable, Monografie Mat. 32, Polish Sci. Publ., Warszawa, 1968.

[18] M. Kuczma, B. Choczewski and R. Ger, Iterative Functional Equations, Encyclopedia Math. Appl. 32, Cambridge Univ. Press, Cambridge, 1990.

[19] W. Lawton, S. L. Lee and Z. Shen, Characterization of compactly supported refinable splines, Adv. Comput. Math. 3 (1995), 137-145.

[20] D. M. Lee, J. G. Lee and S. H. Yoon, A construction of multiresolution analysis by integral equations, Proc. Amer. Math. Soc. 130 (2002), 3555-3563.

[21] S. P. Meyn and R. L. Tweedie, Markov Chains and Stochastic Stability, Springer, London, 1993.

[22] J. Morawiec, On a linear functional equation, Bull. Polish Acad. Sci. Math. 43 (1995), 131-142.

[23] J. Morawiec and L. Reich, On probability distribution solutions of a functional equation, ibid. 53 (2005), 389-399.

[24] - - - The set of probability distribution solutions of a linear functional equation, Ann. Polon. Math. 93 (2008), 253-261.

[25] S. Paganoni Marzegalli, One-parameter system of functional equations, Aequationes Math. 47 (1994), 50-59.

[26] V. Protasov, Refinement equations with nonnegative coefficients, J. Fourier Anal. Appl. 6 (2000), 55-78.

[27] B. Ramachandran and K.-S. Lau, Functional Equations in Probability Theory, Academic Press, Boston, 1991.

[28] C. R. Rao and D. N. Shanbhag, Choquet-Deny Type Functional Equations with Applications to Stochastic Models, Wiley, Chichester, 1994.

[29] W. Vervaat, On a stochastic difference equation and a representation of non-negative infinitely divisible random variables, Adv. Appl. Probab. 11 (1979), 750-783.

Institute of Mathematics

Silesian University

Bankowa 14

40-007 Katowice, Poland

E-mail: t_kochanek@wp.pl

morawiec@ux2.math.us.edu.pl

Received 22.4.2008

and in final form 21.11.2008 